\title{
The Influence and Mechanism of Depression on Memory
}

\author{
Xinyu Jiang ${ }^{1, \uparrow}$, Jiayu Liu ${ }^{2, *, \dagger}$, Ruoyu Song ${ }^{3, \dagger}$ \\ ${ }^{1}$ Wuxi No.1 High School, Wuxi, Jiangsu, China \\ ${ }^{2}$ Beijing Royal School, Beijing, China \\ ${ }^{3}$ The First High School of Changsha, Changsha, Hunan, China \\ *Corresponding author.Email: 20143442@stu.nmu.edu.cn \\ Those authors contributed equally.
}

\begin{abstract}
Depression has been a severe disease that struggles numerous populations and causes problems. Many researches have investigated the effect of depression on memory performance and possible factors behind it, but few combined them together and systematically combed them. Thus, this paper focuses on a more logical relationship among them. We reviewed the previous research in order to show a clearer relationship between memory and depression and corresponding mechanisms of it, which might help the future study of medication and clinician of depression. Researches indicate that those influences are always tend to be negative, show that memory bias and memory destruction are most significant, and depressive patients at age weather old or young all display negative symptoms in memory. At the same time, it shows that hippocampus and dopamine contribute the most. Recent researches are always focus on cognitive bias on depression, while few studies looking for therapies of that. Future study could combine the impact of depression and methods to slow down those symptoms.
\end{abstract}

Keywords: Depression, Memory, Cognitive, Mechanism.

\section{INTRODUCTION}

Depression, also known as major depressive disorder, is a mood disorder that makes you feel constant sadness or lack of interest in life [1]. This definition of depression is essentially syndromal and medical, resembling that of a syndrome in other fields of medicine. This implies a cluster of symptoms and signs which tend to occur together, which are assumed to reflect common pathophysiology, that may not yet be understood, but may have diverse etiologies in different cases. Examples from internal medicine include the malabsorption syndrome and congestive cardiac failure. Life stress is common in various depressive pictures. Dysthymia, a valuable diagnosis, represents a form of what was regarded earlier as neurotic depression. Other subtypes are also discussed [2]. It affects how you feel, think, and behave, and can cause various emotional and physical problems [3]. At the same time, depression can also affect the memory of us.

Researchers believe that depression causes changes in the brain that affect memory. Cortisol disorders, especially in response to stress, are reliably related to the severe and acute manifestations of Major depression disorder, and depression can also affect cortisol [4], too much cortisol in the brain can inhibit the growth of new brain cells in the hippocampus of the brain, causing it to shrink. The hippocampus is very important for the formation of memory. It helps the brain to form, organize and store memories. Therefore, this also suggests that the stress response activated by a depressive episode may affect memory [5]. Depression has been linked to memory problems, such as forgetfulness or confusion. It can also make it difficult to focus on work or other tasks, make decisions, or think clearly. Stress and anxiety can lead to poor memory too. Depression is associated with short-term memory loss [6]. In the other article we notice that authors always arrange the research on the effects of depression, but few of them have conducted research on both neurological and hormonal mechanisms.

Therefore, this paper reviews the effects of depression on memory from the perspectives of neural mechanisms, hormone mechanisms, and age. The effects are talked about in the first part, we introduce that at different ages, depression will have a negative 
impact on memory function. In the next part, the mechanism of depression on memory is introduced. Specifically, depression can make the hippocampus and some of the hormonal change. We can also know that depression is bad for our body at the same time, it can also affect the neurological and hormonal mechanisms of memory.

\section{THE EFFECT OF DEPRESSION ON MEMORY}

\subsection{Memory Bias}

One significant feature of depression is emotion bias, which is related to attention, interpretation, and memory [7]. There is a strong relationship between memory bias and depression [8]. Everaert et al. [9] conducted a partial cueing task, a scrambled sentences task, and a free recall test with 64 participants to study the relationship among attention, interpretation, and memory bias of depression clients. Memory bias was measured as the ratio between the number of negative sentences recalled and the total number of emotional sentences recalled. This study revealed that individuals with more severe depression symptoms tend to focus and approve sentences with negative meanings more frequently than positive ones. His subsequent experiments in 2014 reached similar conclusions [10]. Urban organized a study that asked participants to do a self-report of their emotional experiences and found that it was easier to overestimate negative feelings with participants with depression [11]. It showed that people who had greater risks with recurrent depression would have severer memory bias which would cause them for recalling and amplifying negative emotions.

\subsection{Memory Loss in Depression Patients of Different Ages}

\subsubsection{The Elderly (More than sixty)}

Zhang [12] focused on the short time memory characteristics of the elderly patients with depression. The subjects were divided into 22 depression group (age 66 years old and fluctuate by 3 years) and 42 control group (with similar average ages). The result was that the scores of many different kinds of tests such as pointing memory test, image free recall test, and portrait characteristic association memory test of elderly depression patients were significantly lower than those of the control group. What his conclusion indicated was, the clinical memory scale test of depression patients showed that their memory changes were mainly manifested in the recall test. This may be related to the memory materials or memory test methods. The decline in memory performance of patients with depression may be caused by depression affecting their memory of a certain characteristic material, or depression affecting the ability to recall.

\subsubsection{The Youth (About twenty)}

The Cambridge Neuropsychological Test Automated Battery (CANTAB) has a six-test battery specially designed to assess cognition in studies of depression and associated mood disorders [13].

One of those tests is 'Delayed Matching to Sample'. DMS assessed forced choice recognition memory for non-verbalizable patterns, testing both simultaneous matching and short-term visual memory. This test assessed visual matching ability and visual recognition memory. The participant was shown a complex visual pattern and then after a brief delay, four patterns. The participant must touch the pattern that matches the sample. In some trials the sample and the choice patterns were shown simultaneously, whereas in others there was a delay (of 0,4 or 12 seconds) before the four choices appear. Outcome measures included latency (the participant's speed of response), the number of correct patterns selected and a statistical measure giving the probability of an error after a correct or incorrect response. The results showed that patients presenting with depression often show signs of forgetfulness or memory deficits. DMS is sensitive to these impairments, and can be used to assess changes over time in response to interventions.

Another test is called 'Spatial Working Memory'. SWM looks at how good participants are at retaining and utilising visuospatial information. The test began with a number of coloured squares (boxes) shown on the screen. The aim of this test was that by selecting the boxes and using a process of elimination, the participant should find one yellow 'token' in each of a number of boxes and use them to fill up an empty column on the right-hand side of the screen. Depending on the difficulty level used for this test, the number of boxes could be gradually increased until a maximum of 12 boxes were shown for the participants to search. The colour and position of the boxes used were changed from trial to trial. Outcome measures included errors (selecting boxes that have already been found to be empty and revisiting boxes which have already been found to contain a token). Studies have shown that patients with depression have moderate impairments in working memory [14].

Shelton and Kirwan [15] designed an experiment of young depression patients. The participants were 98 depression patients with an average age of 20 years old. Pattern separation is a mechanism for encoding memories, whereby distinct memory representations are created for very similar stimuli and events. Participants were shown a series of images of everyday objects. Each image belonged to one of three groups, one group of images (foils) only appeared once. A second group of 
images (repeat) appeared twice throughout the study. The final group (lures) consisted of paired images that were visually and conceptually similar. For each image, participants were asked to determine if the image was new, similar, or old. Their results present the mean proportion of "old", "similar", and "new" responses to first, repeated, and lure stimuli for all participants. Participants were very accurate at identifying first and foil image presentations as "new" (96.57\% correct). Accuracy for the repeated images was also high (84.11\%). Responses to the lure images were divided between correctly identifying them as "similar" (54.42\%) and incorrectly calling them "old" (33.76\%). This previous research has demonstrated an association between memory impairments and depression. They concluded that depressed individuals have poorer pattern separation abilities than nondepressed individuals. Furthermore, it is possible that depression is affecting neurogenesis (a suggested mechanism of pattern separation), and this mechanism is responsible for the poor memory performance in depressed individuals.

\section{THE MECHANISMS OF DEPRESSION ON MEMORY}

\subsection{Why Does Age Matter?}

According to the above, different ages of depression all have a certain effect on memory. Although we often associate depression with depression, tiredness, and feelings of hopelessness, what being less known is that some people with depression may have memory problems - for example, feeling more forgetful than usual, so this also causes depression in patients and make more memory loss. Memory problems may appear at the beginning of depression and will persist even if other depressive symptoms improve. Usually, it is our working memory that is affected. This is the short-term memory we use to actively remember things all the time-its problems can make it difficult to concentrate or make decisions. In fact, many cognitive functions are often affected, such as reaction time, attention and planning, decision-making and reasoning. Depression also makes it difficult for our brain to switch between tasks and suppress possible subconscious responses [15], so this is also one of the reasons for memory loss, and why it causes memory loss.

\subsection{Neural Mechanism (Hippocampus)}

Depression is a common neurological disease that seriously affects human health. There are many hypotheses about the pathogenesis of depression, and the most widely recognized and applied is the monoamine hypothesis [16]. As a result, the hippocampus may be one of the important brain regions for memory improvement by novel environment exposure [17] Clinical investigations and neuroimaging data have revealed that the hippocampus volume of depressed people is decreased [18]. Rodent models yielded similar findings. Hippocampal volume is reduced in both unipolar and bipolar depression (BD) patients. Telomere length is positively connected with hippocampus volume, and shorter telomeres are associated with an increased risk of BD [19]. Changes in hippocampal synaptic plasticity are also evident in hippocampus subregions, particularly the CornuAmmonis 3 (CA3) and dentate gyrus, in depression (DG). In this part, we review the link between several hippocampus subregions and LTP and LTD, as well as introducing some antidepressant medications or approaches for treating depression [20].
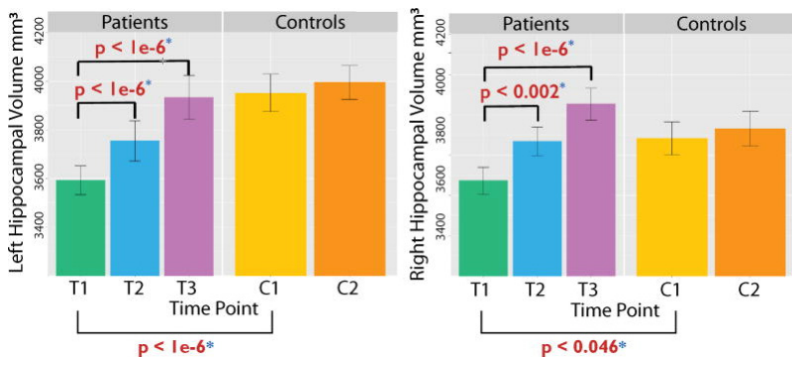

Figure 1. Electroconvulsive therapy (ECT) increases hippocampal volumes bilaterally in patients with major depressive disorder (MDD) [21]

Electroconvulsive therapy (ECT) can increase hippocampal volume in patients with MDD. The destruction of hippocampal neurogenesis in adults with depression has association between depressive symptoms and impaired separation of neutral substance patterns. Conversely, the depression may enhance the pattern separation of the negative material. Therefore, it can be seen from the comparison that depression has a certain effect on the hippocampus.

\subsection{Hormonal Mechanism (Dopamine)}

Anhedonia is considered a core feature of major depressive disorder, and the dopamine system plays a pivotal role in the hedonic deficits described in this disorder. Dopaminergic activity is complex and under the regulation of multiple brain structures, including the ventral subiculum of the hippocampus and the basolateral amygdala [22]. Depression patients take a lot of drugs during their illness, which also affects the secretion and output of dopamine. Chronic treatment with antidepressant drugs potentiates the behavioural stimulant responses elicited by the stimulation of dopamine receptors. Moreover, antidepressants affect dopamine release in several brain areas, so that the potentiation of dopaminergic neurotransmission induced by chronic antidepressant treatments might contribute to their therapeutic effect [23]. 


\section{CONCLUSION}

This paper discussed the effect of depression on memory and its mechanism. Nowadays, more and more people are suffering from depression, at the same time, depression can also cause a series of problems in our brain memory. Through a review of previous literature, we found that depression can cause memory loss and memory decline, which has a negative impact. There are neurological and hormonal mechanisms behind the impact. Specifically, depression not only affects memory, but also changes the hippocampus and dopamine production mechanism. Moreover, memory loss is also one of the common states of depression. Therefore, this article also provides a theoretical basis for the two aspects of the mechanism and impact of depression.

\section{REFERENCES}

[1] Jennifer Casarella, MD on June 28, 2021 https://www.webmd.com/depression/guide/whatis-depression

[2] Eugene S. Paykel, MD, FRCP, FRCPsych, FMedSc 2008 Sep; 10(3): $279 \quad$ - 289. doi: 10.31887/DCNS.2008.10.3/espaykel

[3] By Mayo Clinic Staff Feb. 03, 2018 doi: https:/www.mayoclinic.org/diseasesconditions/depression/symptoms-causes/syc20356007

[4] By L. Sanjay Nandam1 , Matthew Brazel, 1, 2, $\uparrow$ Mei Zhou, $3,4, \uparrow$ and Dhanisha J. Jhaveri 3,4 ,* , Front Psychiatry. 2019; 10: 974.Published online 2020 Jan 22. doi: $10.3389 /$ fpsyt.2019.00974

[5] By Dr. David Woo - January 19, 2021 doi: https:/www.madisonavetms.com/depression-andmemory-loss-does-depression-cause-forgetfulness/

[6] Medically reviewed by Timothy J. Legg, Ph.D., CRNP - Written by Annamarya Scaccia Updated on September 13, 2019 dot: https://www.healthline.com/health/depression/depr ession-and-memory-loss

[7] Linden, S. C., Jackson, M. C., Subramanian, L., Healy, D., \& Linden, D. E. (2011). Sad benefit in face working memory: an emotional bias of melancholic depression. Journal of affective disorders, 135(1-3), $\quad 251 \quad$ - $\quad 257$. https://doi.org/10.1016/j.jad.2011.08.002

[8] Strömgren L. S. (1977). The influence of depression on memory. Acta psychiatrica Scandinavica, 56(2), 109 - 128. https://doi.org/10.1111/j.16000447.1977.tb06670.x
[9] Everaert, J., Tierens, M., Uzieblo, K., \& Koster, E. H. (2013). The indirect effect of attention bias on memory via interpretation bias: evidence for the combined cognitive bias hypothesis in subclinical depression. Cognition \& emotion, 27(8), 14501459.

https://doi.org/10.1080/02699931.2013.787972

[10] Everaert, J., Duyck, W., \& Koster, E. H. (2014). Attention, interpretation, and memory biases in subclinical depression: a proof-of-principle test of the combined cognitive biases hypothesis. Emotion (Washington, D.C.), 14(2), 331-340. https://doi.org/10.1037/a0035250

[11] Urban, E. J., Charles, S. T., Levine, L. J., \& Almeida, D. M. (2018). Depression history and memory bias for specific daily emotions. PloS one, 13(9), $\mathrm{e} 0203574$.

https://doi.org/10.1371/journal.pone.0203574

[12] Zhang Yi-jun, Zhao Yong-guang (Dalian Sanitorium of Shenyang Military Region, PLA, Dalian 116013, China) 'Study on the Short Time Memory Characteristics of the Elderly Patients with Depression' Journal of clinical military medicine (Volume 33, issue 4, August 2005)

[13] University of Cambridge (3 November, 2015) https://www.cambridgecognition.com/blog/entry/6tests-for-assessing-cognition-in-depression

[14] Rock, P. L. et al. 'Cognitive Impairment In Depression: A Systematic Review And MetaAnalysis'. Psychological Medicine 44.10 (2013): 2029-2040. Web. 13 Oct. 2015.

[15] Don J. Shelton, C. Brock Kirwan (Behavioural Brain Research 256 (2013) 20-26)'A possible negative influence of depression on the ability to overcome memory interference 'https://www.sciencedirect.com/science/article/abs/ pii/S0166432813004920 journal homepage: www.elsevier.com/locate/bbr

[16] Cynthia Fu February 9, 2021 10.36pm AEDT doi: https://theconversation.com/people-withdepression-can-sometimes-experience-memoryproblems-heres-why-153392

[17] Wenbo Xu 1, Xiaoxiao Yao 1, Fangyi Zhao 1, Haisheng Zhao 1, Ziqian Cheng 1, Wei Yang 1, Ranji Cui 1, Songbai Xu 2, Bingjin Li 1 Affiliations expand PMID: 33293948PMCID: PMC7718046DOI: 10.1155/2020/8861903

[18] Author links open overlay panelT.AJenkinsa1 EAminaJ.MPearceaM.WBrownbJ.PAggletona Volume 124, Issue 1, 2004, Pages 43-52 
[19] Darren W Roddy 1, Chloe Farrell 2, Kelly Doolin 2, Elena Tozzi 3, Thomas Roman 2, Leonardo O'Keane 2, Erik Frodl 4, Veronica O'Hanlon 5PMID: 30528746 DOI: 10.1016/j.biops ych.2018.08.021

[20] Palmos, A. B., Duarte, R. R. R., Smeeth, D. M., Hedges, E. C., Nixon, D. F., Thuret, S., \& Powell, T. R. (2020). Telomere length and human hippocampal

neurogenesis. Neuropsychopharmacology, 45(13), 2239 - 2247. https://doi.org/10.1038/s41386-02000863-W

[21] Wenbo Xu,1 Xiaoxiao Yao,1 Fangyi Zhao,1 Haisheng Zhao,1 Ziqian Cheng,1 Wei Yang,1 Ranji Cui,1 Songbai Xu,2 and Bingjin Li1 Volume 2020 |Article ID 8861903 | https://doi.org/10.1155/2020/886190 3

[22] Daniel G. Dillon and Diego A. Pizzagalli Trends Neurosci. 2018 Mar; 41(3): 137-149. Published online 2018 Jan 10. doi: 10.1016/j.tins.2017.12.006

[23] Pauline Belujon, $\mathrm{PhD}$ and Anthony A Grace, $\mathrm{PhD}$ Int J Neuropsychopharmacol. 2017 Dec; 20(12): 1036 - 1046.Published online 2017 Jun 29. doi: 10.1093/ijnp/pyx056 